\title{
Factores familiares protectores y de riesgo relacionados al consumo de drogas en adolescentes
}

\author{
Patricia Cid-Monckton ${ }^{1}$ \\ Luiz Jorge Pedrão
}

Se trata de un estudio transversal, cuantitativo, cuyo objetivo fue verificar los factores familiares protectores y de riesgo relacionados al consumo de drogas en adolescentes, considerando los patrones de interacción que se desarrollan en la familia, sus grados de adaptabilidad y vulnerabilidad. Participaron del estudio 80 adolescentes de género femenino, del $1^{\circ}$ al $4^{\circ}$ año de la Enseñanza Media, que respondieron a un cuestionario. Se establecieron los factores de riesgo y protección de mayor relevancia que estarían influyendo en esta situación, tales como, patrones de interacción, grado de adaptabilidad, modo de afrontamiento de los problemas, recursos de la familia y valores. Los factores de mayor riesgo fueron el modo de enfrentar los problemas, y dentro de estos, la falta de apoyo religioso y la búsqueda de apoyo profesional, conjuntamente con las dificultades de comunicación intrafamiliares. Los de menor riesgo fueron los valores, como el esfuerzo personal. Los resultados obtenidos resaltan que el enfermero debe asumir como parte de su rol las intervenciones psicosociales, especialmente en la población escolar, ya que así estarían actuando como agentes de prevención del consumo de drogas.

Descriptores: Control de Riesgo; Conducta del Adolescente; Compuestos Químicos.

\footnotetext{
${ }^{1}$ Profesor Adjunto, Escuela de Enfermería, Universidad de Valparaíso, Valparaíso, Chile. E-mail: pcidmonckton@vtr.net.

${ }^{2}$ Enfermero, Doctor en Enfermería. Profesor Doctor, Escola de Enfermagem de Ribeirão Preto, Universidade de São Paulo, Centro Colaborador de la OMS para el Desarrollo de la Investigación en Enfermería, SP, Brasil. E-mail: lujope@eerp.usp.br.
} 


\section{Fatores protetores e de riscos familiares, relacionados ao consumo de drogas em adolescentes}

Este é um estudo transversal, quantitativo, cujo objetivo foi verificar os fatores protetores e de riscos familiares, relacionados ao consumo de drogas em adolescentes, considerando os padrões de interação que se desenvolvem na família, seus graus de adaptabilidade e vulnerabilidade. Participaram deste estudo 80 adolescentes do sexo feminino, do $1^{\circ}$ ao $4^{\circ}$ ano do ensino médio, que responderam a um questionário. Foram estabelecidos os fatores de risco e de proteção mais relevantes que podem influenciar a situação, como os padrões de interação, grau de adaptabilidade, modo de enfrentamento dos problemas, os recursos da família e valores. Os fatores de maior risco foram o modo de enfrentar os problemas e, dentro desses, a falta de apoio religioso e apoio profissional, juntamente com as dificuldades de comunicação dentro das famílias. Os de menor risco foram os valores, como o esforço pessoal. Os resultados evidenciaram que os enfermeiros devem assumir, como parte de seu papel, as intervenções psicossociais, especialmente entre a população escolar, uma vez que atuariam como agentes na prevenção do uso de drogas.

Descritores: Controle de Risco; Comportamento do Adolescente; Compostos Químicos.

\section{Protective And Family Risk Factors Related To Adolescent Drug Use}

This cross-sectional and quantitative study aimed to verify the family's protective and risk factors related to drugs use in adolescents, considering the interaction patterns developed in the family, their degree of adaptability and vulnerability. Participants in this study were 80 female adolescents, from the $1^{\text {st }}$ to $4^{\text {th }}$ grade of high school, who answered a questionnaire. The most relevant risk and protective factors that would influence the situation were established, such as patterns of interaction, degree of adaptability, way of coping with problems, family resources and values. The major risk factors that emerged were the way people confront problems and, within these, lack of religious support and professional support, besides communication difficulties within families. The lowest risks were values, such as personal effort. The results highlight that nurses should assume psychosocial interventions as part of their role, especially among school-age children as, thus, they would be acting as agents in the prevention of drugs use.

Descriptors: Risk Management; Adolescent Behavior; Chemical Compounds.

\section{Introducción}

Actualmente el uso, abuso y dependencia a las drogas, abarca todos los ámbitos de la convivencia social; su relación con la enfermedad, la violencia, la delincuencia y la pobreza, es ampliamente reconocida. En este sentido la comunidad internacional desarrolla importantes esfuerzos por controlar su producción, comercialización y consumo, además de intentar contener las graves repercusiones que tiene en la salud y su consiguiente costo social. Es así que, el fenómeno de las drogas se hizo competencia de la Salud Internacional, entendiéndose ésta, como la interrelación de diferentes estructuras, procesos y relaciones de ámbito mundial, que inciden en la configuración del proceso salud-enfermedad y en los sistemas de atención. En América Latina este fenómeno se diferencia sólo en las manifestaciones derivadas de los rasgos de identidad cultural característicos, constituyendo de igual manera un grave problema social y de salud pública.

En Chile, se creó en el año de 1990, el Consejo Nacional para el Control de Estupefacientes (CONACE), dependiente del Ministerio del Interior, cuyo objetivo principal era implementar las políticas públicas en torno al problema de las drogas, prevenir el consumo y tráfico de sustancias ilícitas en el país(1). La creciente 
preocupación por las alteraciones de la salud mental llevó al gobierno del país, a través del Ministerio de Salud, a establecer un Programa Nacional de Salud Mental en el año 2000, el que dentro de sus programas, asume el problema del consumo de drogas, como un tema prioritario, especialmente en la población en edad escolar. En Chile, el Consejo Nacional para el Control de Estupefacientes (CONACE), ha realizado varios estudios que validan la situación descrita, e informó entre otros, que la tasa de consumo de tabaco en adolescentes era de $42 \%$, la tasa de consumo de alcohol había aumentado de un 39\% en el año 2003, para 44\% en el año 2005, y la tasa de prevalencia en el uso de drogas especialmente marihuana, había aumentado de 6,5\% en el año 2004 para $8 \%$ en el año 2006 . Ese incremento fue observado específicamente en los niveles educacionales de $3^{\circ}$ y $4^{\circ}$ Enseñanza Media (adolescentes entre 16 y 19 años). En ese mismo estudio, se evaluaron, las relaciones con la familia (actitud parental) frente al uso de las drogas por parte de sus hijos, como factor protector y de riesgo, lo que muestra que al $70 \%$ de los padres les molestaría el consumo de marihuana por parte de sus hijos, y al $40 \%$ el consumo de alcohol.

Por otra parte, se estableció que la falta de compromiso en una relación interpersonal de los padres con los hijos se muestra como un factor que incide notablemente en el consumo de drogas; se encontró una tasa de $10 \%$ de consumo en los hijos con padres involucrados en la relación con estos, versus un 35\% de consumo de drogas en los hijos con padres no involucrados en estas relaciones ${ }^{(2)}$. Lo anterior refuerza el aspecto descrito más adelante, es decir que la familia se reconoce como una de las variables relevantes en el origen y mantenimiento del consumo de drogas. La familia se define, como elemento central en la prevención del consumo de drogas ${ }^{(3)}$.

Dejando de lado otros factores (como los biológicos y genéticos), se establece que los procesos de socialización familiar son importantes al constituir la base predisponente de factores como las actitudes, la personalidad, el auto-concepto, los valores, y las habilidades de comunicación en los adolescentes. En la drogodependencia, la importancia de las variables familiares en el origen y mantenimiento del consumo de sustancias es real; dentro de ellas, se destacan el clima familiar negativo y las variables de comunicación disfuncional de los miembros de la familia. Se establece que estas variables pueden ser evaluadas mediante el constructo de emoción expresada, siendo que los componentes que mostraron mayor relevancia fueron: el criticismo y la hostilidad, tanto en el inicio del consumo, como en la recaída en la drogodependencia(4).

Por otra parte, la familia es considerada como el principal núcleo de socialización de sus integrantes. Es el lugar donde se transmiten valores, se aprenden y adoptan ideas, se adquieren creencias y normas de conductas, y su funcionalidad va a permitir que sus miembros sean personas autónomas, capaces de enfrentarse e integrarse a la vida en sociedad. Sus miembros se agrupan en subsistemas separados por fronteras simbólicas, contribuyendo cada uno al funcionamiento familiar a través del ejercicio de roles y de la realización de tareas necesarias para el conjunto. Se considera entonces a la familia, como un factor moldeador de la personalidad de los adolescentes ${ }^{(5-6)}$.

En Chile, las características de las familias indican que el $94 \%$ de los habitantes vive en algún tipo de situación familiar, pero su conformación es variada; la familia tiende a perder su institucionalización, de 84.000 matrimonios en el año 1980 se ha reducido a 66.600 en el año 2000. Por otra parte el número de hijos ha disminuido de 4,6 hijos por mujer en el año de 1950 a 2,26 en el año de 2002. El número de personas por hogar disminuye de 4 en el año de 1992 para 3,6 en el año de $2002^{(7)}$.

La presente investigación se realizó con adolescentes, por ser esa una etapa de la vida que se caracteriza por el crecimiento y desarrollo humano con una gran vulnerabilidad, donde la búsqueda de identidad personal y grupal hace a los jóvenes inestables, viéndose además influenciados y enfrentados a una diversidad de riesgos psicosociales, como la influencia de los pares para desarrollar conductas sociales y de afrontamiento, que tienen una gran relevancia en su vida de adultos. Por esto, la familia pasa a ser uno de los micro ambientes donde permanece y se desarrolla el adolescente, y es el responsable de lograr que su funcionamiento favorezca un estilo de vida saludable para todos sus miembros. La conducta adolescente es influenciada por los premios y castigos sociales en relación con sus comportamientos, así como por los modelos sociales que rodean al joven ${ }^{(8)}$.

Hay coincidencia según diversos autores que el inicio del consumo de drogas no radica en un factor único, sino que estaría dado por un conjunto de factores que interactúan, determinando así la incidencia y prevalencia de las adicciones. Se ha identificado la existencia de situaciones potencialmente influyentes y/o favorecedoras del consumo de drogas, a las que se les ha denominado factores de riesgo, y otras que 
podrían modificar las acciones de esos factores de riesgo, desestimulando o evitando la aparición de esta problemática, a los que se les ha llamado factores de protección. Entre los factores protectores descritos en la bibliografía tenemos: relación emocional estable al menos con un padre $u$ otro adulto significativo; redes sociales existentes tanto dentro como fuera de la familia; clima educativo abierto, positivo, orientador, con normas y valores claros; modelos sociales que valoren el enfrentamiento positivo de los problemas; equilibrio entre responsabilidades sociales y expectativas de lograr una meta (rendimiento escolar); competencias cognitivas (destrezas de comunicación, empatía); características temperamentales que favorezcan el enfrentamiento efectivo (flexibilidad, capacidad de reflexionar y controlar impulsos, habilidades para comunicarse); experiencias de auto eficacia, confianza en sí mismo y auto concepto positivo; actitud proactiva frente a situaciones estresantes; $y$, experiencia de sentido y significado de la propia vida (fe, religión, coherencia de valores)(6).

En la presente investigación se estudió el funcionamiento familiar, los patrones de interacción con los cuales se desenvuelve la familia, los grados de adaptabilidad y la vulnerabilidad de ellas, con el fin de poder determinar si efectivamente estos factores descritos, estarían actuando como factores protectores y/o de riesgo en el consumo de drogas de sus hijos en edad adolescente.

\section{Objetivo}

Verificar los factores familiares protectores y de riesgos relacionados al consumo de drogas en adolescentes, considerando los patrones de interacción que se desenvuelven en la familia, sus grados de adaptabilidad y vulnerabilidad.

\section{Material y método}

Se trata de una investigación de tipo cuantitativa, transversal con un diseño y análisis descriptivo, aprobada por el Comité de Ética de la Universidad de Valparaíso. El universo estuvo integrado por adolescentes, del género femenino, de $1^{\circ}$ a $4^{\circ}$ año de enseñanza media pertenecientes a un establecimiento de Educación Superior Fiscal de la Quinta Región, en Chile.

El número total de alumnos fue de 400 adolescentes, cuyas edades variaron entre 14 y 19 años. El tipo de muestra fue por conveniencia, no probabilística (interés, facilidades para participar en el estudio, accesibilidad y disponibilidad para la ejecución). El tamaño de la muestra se calculó con el Programa Estadístico, Survey System (Simple Size Calculador, Research Aids, en base a un coeficiente), estableciendo un nivel de confiabilidad de $90 \%$, con un intervalo de confianza de $10 \%$, dando un tamaño de muestreo de 78 alumnas. Se seleccionó un número mayor de adolescentes para la aplicación de encuestas por la posibilidad de pérdidas (90 alumnos), siendo la muestra final de 80 alumnos. Se tomaron al azar 20 alumnos por nivel educacional. Los criterios de inclusión para formar parte de la muestra fueron los siguientes: estudiantes pertenecientes al establecimiento seleccionado y que tuvieran el consentimiento informado firmado por ellos.

Para la recolección de información se usó, el cuestionario "¿Cómo es tú familia?(9) Este instrumento contempla ocho factores que evalúan la adaptabilidad y vulnerabilidad familiar que son: tipo de relaciones, estilo de afrontamiento de los problemas, fuentes de apoyo del adolescente, valores, satisfacción, acumulación de tensiones, problemas de salud y de comportamiento. Los factores descritos se dividen en 19 subfactores. El instrumento fue validado en todos los países que colaboraron en su elaboración (estudio piloto, año $1993)^{(9)}$. La validez de construcción para los factores que constituyen el instrumento, provienen de dos fuentes: las escalas originales de donde fueron tomados, y del análisis estadístico aplicado a los datos obtenidos en el estudio piloto $^{(4)}$. La confiabilidad o consistencia interna de los factores también procede de las mismas fuentes, y de la aplicación del coeficiente Alfa de Cronbach, cuyos resultados oscilan entre 0.51 y 0.81 , lo cual permite la confiabilidad de los distintos factores. Los datos obtenidos en la presente investigación, fueron tratados por medio de estadística descriptiva, estableciéndose el grado de vulnerabilidad, a través del cual se establecen los factores que estarían actuando como protectores o de riesgo, en los adolescentes frente al consumo de drogas. El grado de vulnerabilidad se estableció basándose en la Tabla de Pauta de Calificación de los Cuestionarios, donde según su calificación el factor estudiado estaría actuando como factor de riesgo o como factor protector $^{(4)}$. Los puntajes esperados de vulnerabilidad se dividen en cinco, y para este estudio se estableció una categorización (Tabla 1 ) estableciéndose rangos de porcentaje, para así poder ubicar el resultado y llegar a definir si los factores estudiados, estarían actuando como factores de riesgo o como factores protectores dentro de la población estudiada. 
Tabla 1 - Categorización del grado de Vulnerabilidad en Porcentajes

\begin{tabular}{clc}
\hline Factor & \multicolumn{1}{c}{ Grado de vulnerabilidad } & Porcentaje \\
\hline Riesgo & Muy Alto & $0-19.0$ \\
& Alto & $20-39.9$ \\
& Mediano & $40-59.9$ \\
& Bajo & $60-79.9$ \\
Protector & Muy Bajo & $80-100$ \\
\hline
\end{tabular}

\section{Resultados}

La Tabla 2 muestra los resultados obtenidos, en este estudio para los distintos factores estudiados, en el grado de vulnerabilidad, en sus porcentajes y su clasificación como un factor protector o de riesgo, y de forma secuencial, se presenta de manera descriptiva estos resultados.

Tabla 2 - Vulnerabilidad de los factores estudiados

\begin{tabular}{|c|c|c|c|}
\hline $\begin{array}{c}\text { Grado de } \\
\text { Vulnerabilidad }\end{array}$ & Factores & $\%$ & $\begin{array}{l}\text { Factor de } \\
\text { Riesgo o } \\
\text { Protector }\end{array}$ \\
\hline Muy Alto & Búsqueda Apoyo religioso (A) & 10 & $\begin{array}{c}\text { Factor de } \\
\text { Riesgo }\end{array}$ \\
\hline \multirow[t]{7}{*}{ Alto } & Búsqueda Apoyo Profesional (B) & 19 & \\
\hline & Sentimiento de Felicidad (C) & 25 & \\
\hline & Comunicación Hijo/Padre (D) & 27 & \\
\hline & Acumulación de Tensiones (E) & 33 & \\
\hline & Rendimiento Académico $(F)$ & 34 & \\
\hline & Tipo de Familia (G) & 38 & \\
\hline & Tratamientos $(\mathrm{H})$ & 39 & \\
\hline \multirow[t]{7}{*}{ Mediano } & Búsqueda Apoyo Social (I) & 41 & \\
\hline & Comunicación Padre/Madre- & 45 & \\
\hline & Hijo (D) & 48 & \\
\hline & Poder/Dinero/Sexo $(\mathrm{J})$ & 52 & \\
\hline & Número de Miembros (L) & 58 & \\
\hline & Redefinición del Problema (M) & 58 & \\
\hline & Rutinas Familiares $(\mathrm{N})$ & & \\
\hline \multirow[t]{6}{*}{ Bajo } & Comunicación Hijo/Madre (D) & 63 & \\
\hline & Satisfacción con la Vida (O) & 63 & \\
\hline & Jerarquía y Límites $(P)$ & 65 & \\
\hline & Apoyo del Adolescente (Q) & 71 & \\
\hline & Problemas Salud/ & 79 & \\
\hline & Comportamiento (R) & & \\
\hline \multirow[t]{4}{*}{ Muy Bajo } & Participación (S) & 82 & \\
\hline & Cohesión Familiar (T) & 83 & \\
\hline & Valores Morales (U) & 92 & Factor \\
\hline & Esfuerzo Personal (V) & 97 & Protector \\
\hline
\end{tabular}

El análisis de los factores y subfactores evidencia que con respecto a:

A) Búsqueda de Apoyo Religioso: sólo un 10\% de los encuestados indica que su familia acude a sacerdotes, religiosos o pastores para poder resolver problemas familiares, por lo que este subfactor sería considerado de alta vulnerabilidad y de riesgo.
B) Búsqueda de Apoyo Profesional: un 19\% indica que su familia ha buscado la ayuda de profesionales, ya sean abogados, médicos, psicólogos u otros como ayuda para resolver sus problemas, mientras que sólo un $4 \%$ de estos, indica que su familia utiliza programas que existen en la comunidad para ayudar a personas que tiene dificultades, con lo cual este subfactor sería de alta vulnerabilidad.

C) Sentimientos de Felicidad: los adolescentes se sienten tan felices como la mayoría de sus grupos de pares en un $48 \%$, un $34 \%$ se considera de los más felices o más que la mayoría, y un $13 \%$ menos feliz que la mayoría y sólo un $6 \%$ se considera uno de los menos felices. Lo anterior nos muestra este factor como de alta vulnerabilidad y por lo tanto sería un factor de riesgo.

D) Comunicación Hijos/Padres (Comunicación Padre/ Madre/Hijo - Comunicación Hijo Padre - Comunicación Hijo/Madre): un 63,25\% de las encuestadas respondió que la comunicación con su madre era buena, siendo más fácil y existiendo un mayor grado de apertura, mientras que con el padre sólo un $27 \%$ contestó lo mismo, por lo que este subfactor se consideraría de alta vulnerabilidad.

E) Acumulación de Tensiones: ítem que contempla 17 aspectos: el $70 \%$ de los adolescentes contestó que en su familia no había ocurrido ninguna situación difícil durante el último año; mientras que un 9,5\% indicó que habían ocurrido, pero no afectó a su familia, y un $20,4 \%$ que habían ocurrido y que afectaron a su familia. Los factores que más afectaron a la familia de los adolescentes fueron, los problemas de dinero en un $47 \%$ de los casos, la muerte de un miembro de la familia, un pariente 0 un amigo cercano, con un $46 \%$, y hogares donde hubo serios conflictos o problemas familiares con un $34 \%$. Los resultados nos muestran una alta vulnerabilidad por lo que estaría constituyendo un factor de riesgo.

F) Rendimiento Académico del Adolescente: el 44\% siente que su rendimiento es igual que el de la mayoría, un $14 \%$ que su rendimiento es un poco peor que el de la mayoría y un $43 \%$ considera que su rendimiento en relación a sus compañeros, es un poco mejor y mucho mejor que el de la mayoría. Estos resultados hacen que éste factor sea de alta vulnerabilidad.

G) Tipo de Familia: en el $29 \%$ de los casos la familia era completa, en el $9 \%$ era extensa, en el $18 \%$ sólo vivían con la madre y en $9 \%$ de los casos conformaban otro tipo de familia. En este caso sería un factor alta vulnerabilidad, porque la familia completa se considera como factor protector. 
H) Tratamientos - Problemas de Salud Comprendidos en el Ámbito de la Salud Mental: el de mayor recurrencia fue el tratamiento psicológico o psiquiátrico con $53 \%$ de los adolescentes, siguiéndolo los tratamientos por heridas de peleas o riñas con un $8 \%$, lo cual nos indica la presencia de síntomas y dificultades que comprometen la salud de los adolescentes. El factor sería de vulnerabilidad alta, constituyendo un factor de riesgo.

I) Búsqueda de Apoyo Social: los adolescentes indican que cuando han tenido problemas dentro de su núcleo familiar, $41 \%$ de las veces acuden buscando ayuda de amigos, mientras que sólo $15 \%$ se acerca a personas que hubiesen tenido un problema similar al de ellos. Este factor sería de mediana vulnerabilidad.

J) Poder/Dinero y Sexo: lo que tiene más importancia en la vida de los adolescentes encuestados, es ejercer influencia sobre los demás en $60 \%$ de los casos, ya sea en los estudios, en el trabajo o en la comunidad. Tener dinero, se considera como la segunda prioridad con $56 \%$ y, finalmente, sólo $28 \%$ del grupo en estudio indica que tener una vida sexual activa es valioso para la vida. Evaluado el ítem en su totalidad, nos da una vulnerabilidad media, destacándose, pese a esto último, la cantidad de adolescentes con una vida sexual activa.

L) Número de Miembros de la Familia: 52\% de los encuestados indicó que en su familia viven entre 2 a 4 personas, mientras que sólo el $4 \%$ de indica que su familia es conformada por 8 y más de 9 miembros, por tanto en nuestro grupo estudiado sería un factor de mediana vulnerabilidad. El 59\% de los adolescentes tenía entre 3 y 6 hermanos lo que actuaría como factor protector.

M) Redefinición del Problema: 58\% de los adolescentes indicó que su familia tiene los recursos necesarios para hacer frente a los inconvenientes que se le presentan, siendo este subfactor por lo tanto, de mediana vulnerabilidad.

N) Rutinas Familiares: de acuerdo con las respuestas, la madre dedica más tiempo que el padre para hablar con sus hijos, $77 \%$ y $39 \%$ respectivamente. Con respecto a compartir las comidas, compartir alguna actividad diaria y efectuar alguna actividad en familia, el 57,2\% de los adolescentes lo realiza, lo que indicaría que más de la mitad de los encuestados concibe un alto grado de estructuración y de organización de la vida familiar, otorgándole a este subfactor un grado de vulnerabilidad medio.

O) Satisfacción con la Vida: de los 9 ítems que conforman este subfactor, sólo en uno de ellos los adolescentes obtuvieron una baja puntuación, es el que está relacionado con el rendimiento escolar, el $47 \%$ no lo considera tan importante. En los otros 8 ítems, los adolescentes muestran un buen grado de satisfacción consigo mismo (64\%), con los amigos $77 \%$, con la vida religiosa de la familia $52 \%$, con los servicios de salud que dispone la familia $61 \%$, con el barrio o comunidad donde vive $64 \%$, con la situación económica de la familia $53 \%$, con el trabajo u ocupación principal $68 \%$, y con la familia $77 \%$. Los resultados nos indicarían este factor como de vulnerabilidad baja.

P) Jerarquía y Límites: el 85\% de los encuestados indicó tener bien claro quién manda en su familia y sólo un $7 \%$ indican no tener claridad. El 44\% de los adolescentes asume como autoridad a ambos padres, mientras que el $35 \%$ le otorga este rol a la madre y sólo el $2 \%$ al padre. Se muestra así que este factor es de baja vulnerabilidad, por lo que estaría en este caso actuando más bien como factor protector.

Q) Fuentes de Apoyo del Adolescente: él siente que cuenta con el apoyo de sus padres en el $65 \%$ de los casos y de sus amigos personales en el $77 \%$, y son a los cuales recurren si tiene algún problema, por lo que este factor sería de baja vulnerabilidad.

R) Problemas de Salud y Comportamiento: el 21\% expresa que ha tenido algún problema, por lo que se concluye que el nivel de adaptación del adolescente y de su familia es adecuado, el factor que muestra una mayor incidencia fue la preocupación del aspecto físico, 19\%, siguiéndole el sentirse sólo o nervioso todo el tiempo con un $14 \%$, lo que nos daría una vulnerabilidad baja para éste ítem.

S) Participación en la Solución de Problemas Familiares: el $79 \%$ de los adolescentes encuentra que sus padres consideran sus ideas en la solución de estos problemas y $84 \%$ de ellos considera que la familia enfrenta los problemas unidos, lo cual nos muestra un grado de vulnerabilidad bajo.

T) Cohesión Familiar: el 83,6\%, de los adolescentes encuestados indicó que tiene un alto nivel de cohesión familiar, un fuerte apego emocional y que en su familia existe una fuerte expresión de afecto entre los miembros, mientras que el $17,4 \%$, restante, obtuvo puntajes bajos en su respuesta, lo que indica que existe un desligamiento afectivo en el entorno familiar, que serían los más vulnerables; con lo cual este subfactor sería de muy baja vulnerabilidad en nuestro estudio.

U) Valores Morales: las respuestas entregadas indican que el $92 \%$ considera los siguientes aspectos definitivos para vivir, tener una familia unida, ser respetado, 
respetar a los demás, ser honesto, creer en un ser superior. La creencia en Dios o un Ser Superior, es el que tiene el menor de porcentaje (72\%); mientras que ser una persona honesta y recta es para todos los adolescentes definitivo (100\%), por lo que este factor muestra una vulnerabilidad muy baja en nuestro grupo de estudio y estaría actuando como factor protector.

v) Esfuerzo Personal: el $97 \%$ de los encuestados expresó que alcanzar un alto nivel de estudios es muy importante en su vida, y el $98 \%$ de ellos, indica que realizarse en el trabajo por ellos escogido, es de gran importancia. Los resultados nos muestran un muy bajo grado de vulnerabilidad con respecto a éste tema.

\section{Discusión}

Los resultados obtenidos coinciden con los observados en la literatura, en la cual la religiosidad y la plegaria se correlacionan de forma estadísticamente positiva, con la satisfacción con la propia vida y con mejores logros personales(10). En una revisión sobre salud mental y religión, un porcentaje importante de estudios mostró correlaciones positivas entre ambos elementos $^{(11)}$.

La familia se define como elemento central en la prevención del consumo de drogas y los procesos de socialización familiar son importantes, pues constituyen la base de predisponentes como son las actitudes, la personalidad, el autoconcepto, los valores, y las habilidades de comunicación, en los adolescentes ${ }^{(12)}$.

Las variables del ámbito familiar que más frecuentemente se relacionan con el consumo de drogas por parte de un miembro de la familia, son, un clima familiar negativo (estrés, negatividad, rechazo), un conflicto familiar excesivo (hostilidad), exceso protectores y falta de comunicación adecuada(4). Por otra parte, se señala también que es menos probable que los hijos de familias altamente cohesionadas que recibieran ofertas de tabaco y alcohol, probaran o consumieran estas sustancias, se embriagaran o tuvieran intención de fumar o beber en un futuro(13), lo que también se comprueba en esta investigación.

Considerando que las variables familiares pueden ser pronosticadores significativos del consumo de drogas, es posible deducir que las dimensiones relacionadas con la existencia de conflictos entre el adolescente y sus padres, y el consumo familiar de drogas son las que predicen un mayor riesgo de que el joven se implique en el uso de cualquier tipo de sustancia(11,14-15), lo que quedó manifestado en los resultados encontrados.

\section{Conclusiones}

De ésta investigación se puede concluir que los factores de mayor riesgo fueron el modo de enfrentar los problemas y dentro de él la falta de apoyo religioso, las dificultades de comunicación intrafamiliar, el tipo y características de la familia; y los factores de menor riesgo fueron los valores, como el esfuerzo personal.

Es importante destacar el espacio profesional para enfermería en el área de la prevención del consumo de drogas, que se confirma con ésta investigación efectuada en el ámbito de la educación, donde se puede trabajar reforzando y estimulando las actitudes y aspectos de la personalidad conducentes a evitar el consumo de drogas, como son el autoconocimiento, la autoestima, la comunicación y la resiliencia. Dado que en éste trabajo la población estudiada es representativa, los resultados se utilizarán para planificar estrategias de intervención dentro del establecimiento educacional, basadas específicamente en reforzar los factores protectores identificados y gestionar con los padres, profesorado y organizaciones de la comunidad intervenciones dirigidas a potenciar los factores de protección, y a reducir los factores de riesgo mencionados como resultado de esta investigación. Entre ellos se destaca la importancia de la comunicación dentro de la familia y el rendimiento académico, entre otros.

\section{Agradecimientos}

Agradecemos a la Comisión Interamericana para el Control del Abuso de Drogas/CICAD de la Secretaria de Seguridad Multidimensional/SSM de la Organización de los Estados Americanos/OEA, la Secretaria Nacional de Políticas sobre Drogas/SENAD do Gabinete de Seguridad Institucional/Brasil, la Escuela de Enfermería de Ribeirao Preto de la Universidad de Sao Paulo y Centro Colaborador de la Organización Mundial de la Salud para el Desarrollo de la Investigación en Enfermería, la población representada en los estudios de investigación, bien como a las autoridades de las universidades representadas por los participantes del Programa En-Line de Especialización en Investigación sobre el Fenómeno de las Drogas, periodos 2006, 2007, 2008 y 2009.

\section{Referencias}

1. Consejo Nacional para el Control de Estupefaciente. (CONACE). Sexto Estudio de Drogas en Población General. 2004. Gobierno de Chile; 2005. 
2. Consejo Nacional para el Control de Estupefacientes (CONACE). Sexto Estudio Nacional de Drogas en Población Escolar de Chile $8^{\circ}$ Básico a $4^{\circ}$ Medio 2005. Gobierno de Chile; 2006.

3. Minuchin S. Familias y Terapia familiar. Barcelona: Edit. Gedisa; 1979.

4. Becerra JA. Variables familiares y drogodependencia: la influencia de los componentes de la emoción expresada. 2008. Psicología Científica.com. [acceso 15 nov 2008]. Disponible en: http://www.psicologiacientifica.com/bv/ imprimir-344-variables-familiares-y-drogodependenciala-influencia-de-los-componentes-de-la-emocionexpresada.html.

5. Muñoz-Rivas MJ, López JLG. Factores familiares de riesgo y de protección para el consumo de drogas en adolescentes. Psicothema 2000;13(1):87-94.

6. Alba MRCde, Lloret PB, Lloret FB. Implicación en los comportamientos de agonismo y ajuste social al grupo de iguales en niños de cuatro y cinco años. Psicothema 2001;13(2):258-62.

7. Censo 2002, Chile. Instituto Nacional de Estadísticas (INE). [acceso:10 out 2008]. Disponible en: http:// www.ine.cl/cd2002/sintesiscensal.pdf

8. Abarca N, Errázuriz M. Transformaciones de la Familia Chilena. Propuestas para la conciliación Trabajo y Familia.2005. Universidad Diego Portales, Instituto de Ciencias Sociales. [acceso 20 out 2008]. Disponible en: http://vinculosconlasociedad.uc.cl/documentos/71.pdf

9. Hernández A, Beuhring T, Berner E, Blue R, Florenzano $R$, Lammers $C$, et al. Familia y Adolescencia: Indicadores de Salud. Manual de Aplicación de Instrumentos. Washington (D.C): I.Kellog; 1996.

10. Peñafiel E. Factores de riesgo y protección en el consumo de sustancias en adolescentes, Pulso Rev Educ. 2009;32:147-73.

11. Vanistandael S. Resiliency and Spiritual Life. ICCB News, Ginebra, 1995.

12. Lloret D. Alcoholismo: Una visión familiar. Salud y Drogas. Instituto de Investigación de Drogodependencias 2001;1(1):113-28.

13. Florenzano U, Ramón E. Adolescente y sus conductas de riesgo.Chile: Edit. Universidad Católica de Chile; 1998.

14. Rutter M. Pathways from childhood to Adult Life. J Child Psychol Psychiatry 1989;30:23-51.

15. Muñoz-Rivas MJ, López JLG. Factores familiares de riesgo y de protección para el consumo de drogas en

Recibido: 8.12.2009 adolescentes, Psicothema 2001;13(1):87-94.

Aceptado: 17.9.2010 